\title{
Multiple Solutions for a Second-Order Impulsive Sturm-Liouville Equation
}

\author{
Jingli Xie ${ }^{1,2}$ and Zhiguo Luo ${ }^{2}$ \\ ${ }^{1}$ College of Mathematics and Statistics, Jishou University, Jishou, Hunan 416000, China \\ ${ }^{2}$ Department of Mathematics, Hunan Normal University, Changsha, Hunan 410081, China \\ Correspondence should be addressed to Zhiguo Luo; luozg@hunnu.edu.cn
}

Received 29 March 2013; Revised 27 May 2013; Accepted 18 June 2013

Academic Editor: Gennaro Infante

Copyright (c) $2013 \mathrm{~J}$. Xie and Z. Luo. This is an open access article distributed under the Creative Commons Attribution License, which permits unrestricted use, distribution, and reproduction in any medium, provided the original work is properly cited.

\begin{abstract}
We study the existence of solutions to a boundary value problem of a second-order impulsive Sturm-Liouville equation with a control parameter $\lambda$. By employing some existing critical point theorems, we find the range of the control parameter in which the boundary value problem admits at least three solutions. It is also shown that, under certain conditions, there exists an interval of the control parameter in which the boundary value problem possesses infinitely many solutions. Some examples are given to demonstrate the main results in this paper.
\end{abstract}

\section{Introduction}

The aim of this paper is to investigate the existence of multiple solutions of the following Neumann boundary value problem with impulsive Sturm-Liouville type equation:

$$
\begin{gathered}
-\left(p(t) u(t)^{\prime}\right)^{\prime}+q(t) u(t)=\lambda f(t, u(t)), \quad t \neq t_{j}, t \in[0,1], \\
\Delta\left(p\left(t_{j}\right) u^{\prime}\left(t_{j}\right)\right)=I_{j}\left(u\left(t_{j}\right)\right), \quad j=1,2, \ldots, m, \\
u^{\prime}(0)=u^{\prime}(1)=0,
\end{gathered}
$$

where $p \in C^{1}\left([0,1], q \in C([0,1]), f \in C([0,1] \times R, R), I_{j} \in\right.$ $C(R, R), 0=t_{0}<t_{1}<t_{2}<\cdots<t_{m}<t_{m+1}=1$, and $\lambda$ is a positive real parameter. $\Delta u^{\prime}\left(t_{j}\right)=u^{\prime}\left(t_{j}^{+}\right)-u^{\prime}\left(t_{j}^{-}\right)$, where $u^{\prime}\left(t_{j}^{+}\right)$and $u^{\prime}\left(t_{j}^{-}\right)$denote the right and left limits, respectively, of $u^{\prime}(t)$ at $t=t_{j}, j=1,2, \ldots, m$.

In the last few years, the existence of multiple solutions to Neumann problems has been widely investigated [1-5]. But little research has focused on the existence of multiple solutions for impulsive Sturm-Liouville equations whose right-hand side nonlinear term is depending on a parameter $\lambda$. Processes subject to sudden changes in their states are modeled by the impulsive differential equations and have been investigated in various fields of science and technology. In the motion of spacecraft, one has to consider instantaneous impulses at a position with jump discontinuities in velocity, but no change in the position $[6,7]$. This motivates us to consider problem (1).

In the literature, tools employed to establish the existence of solutions of impulsive differential equations include fixed point theorems, the upper and lower solutions method, the degree theory, critical point theory, and variational methods. See, for example, [8-20]. In this paper, our focus is on the existence of solutions of problem (1) with $\lambda$ being the parameter. The problem is first transformed into the existence of critical points of some variational structure. Then with the help of critical point theory, results on the existence of at least three solutions and infinitely many solutions are established.

The rest of this paper is organized as follows. In Section 2 we present some preliminary results. Our main results and their proofs are given in Section 3.

\section{Preliminaries}

Throughout we assume that $p(t)$ and $q(t)$ satisfy

$$
p_{0}=\min _{t \in[0,1]} p(t)>0, \quad q_{0}=\min _{t \in[0,1]} q(t)>0 .
$$


Take $X=W^{1,2}([0,1])$ and define

$$
\|u\|_{X}=\left(\int_{0}^{1}\left(p(t)\left|u^{\prime}(t)\right|^{2}+q(t)|u(t)|^{2}\right) d t\right)^{1 / 2}
$$$$
u \in X
$$

For the norm in $C([0,1])$, we put

$$
\|u\|_{\infty}=\max _{t \in[0,1]}|u(t)| .
$$

We have the following relation.

Lemma 1. Let $M_{1}=\min \left\{p_{0}, q_{0}\right\}$. Then

$$
\|u\|_{\infty} \leq \sqrt{\frac{2}{M_{1}}}\|u\|_{X}, \quad \forall u \in X .
$$

Proof. For any $u \in X$, it follows from the mean value theorem that

$$
\int_{0}^{1} u(s) d s=u(\tau)
$$

for some $\tau \in[0,1]$. Hence, for $t \in[0,1]$, using Hölder inequality and (2), we have

$$
\begin{aligned}
|u(t)|= & \left|u(\tau)+\int_{\tau}^{t} u^{\prime}(s) d s\right| \leq|u(\tau)|+\int_{0}^{1}\left|u^{\prime}(s)\right| d s \\
\leq & \int_{0}^{1}|u(s)| d s+\int_{0}^{1}\left|u^{\prime}(s)\right| d s \\
\leq & \left(\frac{1}{q_{0}}\right)^{1 / 2}\left(\int_{0}^{1} q(t)|u(t)|^{2} d t\right)^{1 / 2} \\
& +\left(\frac{1}{p_{0}}\right)^{1 / 2}\left(\int_{0}^{1} p(t)\left|u^{\prime}(t)\right|^{2} d t\right)^{1 / 2} \\
\leq & \sqrt{\frac{2}{M_{1}}}\|u\|_{X} .
\end{aligned}
$$

Define a functional $\varphi_{\lambda}$ as

$$
\varphi_{\lambda}(u)=\Phi(u)-\lambda \Psi(u), \quad u \in X,
$$

where

$$
\begin{gathered}
\Phi(u)=\frac{1}{2}\|u\|_{X}^{2}+\sum_{j=1}^{m} \int_{0}^{u\left(t_{j}\right)} I_{j}(s) d s \\
\Psi(u)=\int_{0}^{1} F(t, u) d t
\end{gathered}
$$

with

$$
F(t, u)=\int_{0}^{u(t)} f(t, s) d s
$$

Note that $\varphi_{\lambda}$ is Fréchet differentiable at any $u \in X$, and for any $v \in X$, we have

$$
\begin{aligned}
\varphi_{\lambda}^{\prime}(u)(v)= & \lim _{h \rightarrow 0} \frac{\varphi_{\lambda}(u+h v)-\varphi_{\lambda}(u)}{h} \\
= & \int_{0}^{1}\left(p(t) u^{\prime}(t) v^{\prime}(t)+q(t) u(t) v(t)\right) d t \\
& +\sum_{j=1}^{m} I_{j}\left(u\left(t_{j}\right)\right) v\left(t_{j}\right) \\
& -\lambda \int_{0}^{1} f(t, u(t)) v(t) d t .
\end{aligned}
$$

Lemma 2. If $u \in X$ is a weak solution of problem (1), then $u$ is a classical solution of problem (1).

Proof. For any function $v \in X$, we get

$$
\begin{aligned}
0= & \int_{0}^{1}\left(p(t) u^{\prime}(t) v^{\prime}(t)+q(t) u(t) v(t)\right) d t \\
& +\sum_{j=1}^{m} I_{j}\left(u\left(t_{j}\right)\right) v\left(t_{j}\right) .
\end{aligned}
$$

By the regularity theory, the weak solution $u$ is a classical solution of problem (1).

Next we show that a critical point of the functional $\varphi_{\lambda}$ is a solution of problem (1).

Lemma 3. If $u \in X$ is a critical point of $\varphi_{\lambda}$, then $u$ is a classical solution of problem (1).

Proof. If $u \in X$ is a critical point of $\varphi_{\lambda}$, by (12) and Lemma 2, we have $u$ is a classical solution of problem (1)

For $r \in \mathbb{R}$, we define

$$
\begin{array}{r}
\rho(r)=\inf _{v \in \Phi^{-1}((-\infty, r))} \frac{\sup _{u \in \Phi^{-1}((-\infty, r))} \Psi(u)-\Psi(v)}{r-\Phi(v)}, \\
\gamma_{1}:=\liminf _{r \rightarrow+\infty} \rho(r), \quad \delta:=\liminf _{r \rightarrow\left(\inf _{X} \Phi\right)^{+}} \rho(r) .
\end{array}
$$

\section{Main Results}

3.1. Existence of At Least Three Solutions. In this section we derive conditions under which problem (1) admits at least three solutions. For this purpose, we introduce the following assumptions.

(H1) Assume that there exists a positive constant $k_{1}$ such that for each $u \in X$

$$
0 \leq \sum_{j=1}^{m} \int_{0}^{u\left(t_{j}\right)} I_{j}(s) d s \leq k_{1} \max _{j \in\{1,2, \ldots, m\}}\left|u\left(t_{j}\right)\right|^{2} .
$$


(H2) Assume that there exist positive constants $a, b$, and $\gamma \in[0,1)$, such that

$0<f(t, u) \leq a+b|u|^{\gamma}, \quad$ for $(t, u) \in[0,1] \times \mathbb{R}$.

Let $k_{0}=\int_{0}^{1} q(t) d t$ and let $k_{2}=(1 / 2) k_{0}+k_{1}$ with $k_{1}$ given in (16). Clearly, $M_{1} \leq k_{0}$. For constants $c_{1}, c$, we define

$$
\begin{gathered}
\lambda_{1}=\frac{4}{M_{1}} \frac{\int_{0}^{1} \max _{|u| \leq c_{1}} F(t, u) d t}{c_{1}^{2}}, \\
\lambda_{2}=\frac{2}{3} \frac{\int_{0}^{1} F(t, c) d t .}{k_{2} c^{2}} .
\end{gathered}
$$

Theorem 4. Assume that (H1), (H2) are satisfied. If there exist two positive constants $c_{1}, c$ satisfying $c_{1}<c$, and

$$
\lambda_{1}<\lambda_{2}
$$

then, for each $\lambda \in\left(1 / \lambda_{2}, 1 / \lambda_{1}\right)$, problem (1) admits at least three solutions.

Proof. By Lemma 3, it suffices to show that the functional $\varphi_{\lambda}$ defined in (8) has at least three critical points. We prove this by verifying the conditions given in [21, Theorem 3.2]. Note that $\Phi$ defined in (9) is a nonnegative Gâteaux differentiable, coercive, and sequentially weakly lower semicontinuous functional and its Gâteaux derivative admits a continuous inverse on $X^{*}$. Moreover, $\Psi$ defined in (10) is a continuously Gâteaux differentiable functional whose Gâteaux derivative is compact. Set

$$
r=\frac{M_{1} c_{1}^{2}}{4} .
$$

Note that $u_{1}(t)=c \in X$. It then follows from (H1) that

$$
\begin{aligned}
\Phi\left(u_{1}\right) & =\frac{1}{2}\left\|u_{1}\right\|_{X}^{2}+\sum_{j=1}^{m} \int_{0}^{u_{1}\left(t_{j}\right)} I_{j}(s) d s \\
& =\frac{1}{2} c^{2} \int_{0}^{1} q(s) d s+\sum_{j=1}^{m} \int_{0}^{c} I_{j}(s) d s \\
& \leq \frac{1}{2} k_{0} c^{2}+k_{1} c^{2} \\
& \leq k_{2} c^{2}, \\
\Phi & \left(u_{1}\right) \geq \frac{1}{2}\left\|u_{1}\right\|_{X}^{2}=\frac{1}{2} k_{0} c^{2} .
\end{aligned}
$$

Then, we have

$$
2 r=\frac{M_{1} c_{1}^{2}}{2}<\frac{1}{2} k_{0} c^{2} \leq \Phi\left(u_{1}\right) .
$$

For $u \in X$ satisfying $\Phi(u)<r$, by Lemma 1 , one has

$$
\begin{array}{r}
|u|^{2} \leq\|u\|_{\infty}^{2} \leq \frac{2}{M_{1}}\|u\|_{X}^{2} \leq \frac{4}{M_{1}} \Phi(u)<\frac{4}{M_{1}} r=c_{1}^{2}, \\
t \in[0,1],
\end{array}
$$

which implies that

$$
\Psi(u)=\int_{0}^{1} F(t, u(t)) d t \leq \int_{0}^{1} \max _{|u| \leq c_{1}} F(t, u) d t .
$$

Hence

$$
\sup _{u \in \Phi^{-1}((-\infty, r))} \Psi(u) \leq \int_{0}^{1} \max _{|u| \leq c_{1}} F(t, u) d t .
$$

So, one has

$$
\begin{gathered}
0 \leq \frac{\sup _{u \in \Phi^{-1}((-\infty, r))} \Psi(u)}{r} \leq \frac{4}{M_{1}} \frac{\int_{0}^{1} \max _{|u| \leq c_{1}} F(t, u) d t}{c_{1}^{2}}, \\
\frac{2}{3} \frac{\Psi\left(u_{1}\right)}{\Phi\left(u_{1}\right)} \geq \frac{2}{3} \frac{\int_{0}^{1} F(t, c) d t .}{k_{2} c^{2}} .
\end{gathered}
$$

Making use of (19), we obtain

$$
\frac{\sup _{u \in \Phi^{-1}((-\infty, r))} \Psi(u)}{r}<\frac{2}{3} \frac{\Psi\left(u_{1}\right)}{\Phi\left(u_{1}\right)} .
$$

By (H1) and (H2), when $\lambda \in\left(1 / \lambda_{2}, 1 / \lambda_{1}\right)$, we easily obtain that the functional $\varphi_{\lambda}$ is coercive.

Thus all conditions in [21, Theorem 3.2] are verified, and hence for each $\lambda \in\left(1 / \lambda_{2}, 1 / \lambda_{1}\right)$, the functional $\varphi_{\lambda}=\Phi-\lambda \Psi$ admits at least three critical points. Consequently, problem (1) admits at least three solutions.

Example 5. Consider the boundary value problem

$$
\begin{gathered}
-\left(e^{t} u^{\prime}(t)\right)^{\prime}+u(t)=\lambda f(t, u), \quad t \neq t_{1}, t \in[0,1], \\
\Delta\left(e^{t_{1}} u^{\prime}\left(t_{1}\right)\right)=u\left(t_{1}\right), \quad t_{1}=\frac{1}{2}, \\
u^{\prime}(0)=u^{\prime}(1)=0,
\end{gathered}
$$

where

$$
f(t, u)= \begin{cases}e^{u}, & u \leq 16, t \in[0,1], \\ u^{1 / 2}+e^{16}-4, & u>16, t \in[0,1] .\end{cases}
$$

Here, $I_{1}(s)=s, p(t)=e^{t}, q(t)=1$, and $m=1$. Note that (H1), (H2) are satisfied. Moreover, we have $k_{0}=1, k_{1}=1 / 2$, $k_{2}=1, M_{1}=1$, and

$$
F(t, u)=\left\{\begin{aligned}
e^{u}-1, & u \leq 16, t \in[0,1], \\
\frac{2}{3} u^{3 / 2}+\left(e^{16}-4\right) u & \\
+\frac{61}{3}-15 e^{16}, & u>16, t \in[0,1] .
\end{aligned}\right.
$$

Choose $c_{1}=1, c=16$. Direct calculations give

$$
\begin{aligned}
& \lambda_{1}=\frac{4}{M_{1}} \frac{\int_{0}^{1} \max _{|u| \leq c_{1}} F(t, u) d t}{c_{1}^{2}}=4(e-1)<8, \\
& \lambda_{2}=\frac{2}{3} \frac{\int_{0}^{1} F(t, c) d t .}{k_{2} c^{2}}=\frac{1}{384}\left(e^{16}-1\right)>100 .
\end{aligned}
$$

Therefore it follows from Theorem 4 that (28) admits at least three solutions in $X$ provided that $\lambda \in(0.01,0.125)$. 
3.2. Existence of Infinitely Many Solutions. In this section, we derive some conditions under which problem (1) admits infinitely many distinct solutions. To this end, we need the following assumption.

(H3) Assume that

$$
F(t, u) \geq 0, \quad(t, u) \in[0,1] \times \mathbb{R} .
$$

Set

$$
\begin{gathered}
\mu_{1}=\frac{4}{M_{1}} \liminf _{\xi \rightarrow+\infty} \frac{\int_{0}^{1} \max _{|u| \leq \xi} F(t, u) d t}{\xi^{2}} \\
\mu_{2}=\frac{1}{k_{2}} \limsup _{\xi \rightarrow+\infty} \frac{\int_{0}^{1} F(t, \xi) d t}{\xi^{2}} .
\end{gathered}
$$

Theorem 6. Assume that (H3) is satisfied. If

$$
\mu_{1}<\mu_{2}
$$

holds, then for each $\lambda \in\left(1 / \mu_{2}, 1 / \mu_{1}\right)$, problem (1) has an unbounded sequence of solutions in $X$.

Proof. We apply [22, Theorem 2.1] to show that the functional $\varphi_{\lambda}$ defined in (8) has an unbounded sequence of critical points.

We first show that $\gamma_{1}<+\infty$. Let $\left\{\xi_{n}\right\}$ be a sequence of positive numbers such that $\xi_{n} \rightarrow+\infty$ as $n \rightarrow \infty$ and

$$
\lim _{n \rightarrow+\infty} \frac{\int_{0}^{1} \max _{|u| \leq \xi_{n}} F(t, u) d t}{\xi_{n}^{2}}=\liminf _{\xi \rightarrow+\infty} \frac{\int_{0}^{1} \max _{|u| \leq \xi} F(t, \xi) d t}{\xi^{2}} .
$$

For any positive integer $n$, we let $r_{n}=M_{1} \xi_{n}^{2} / 4$. For $u \in X$ satisfying $\Phi(u)<r_{n}$, similar to the proof of Theorem 4 , one can show that

$$
\|u\|_{\infty}^{2} \leq \frac{4}{M_{1}} \Phi(u)<\xi_{n}^{2}, \quad t \in[0,1],
$$

which implies that

$$
\Psi(u)=\int_{0}^{1} F(t, u) d t \leq \int_{0}^{1} \max _{|u| \leq \xi_{n}} F(t, u) d t .
$$

Note that $\Psi(0)=\Phi(0)=0$; thus we have

$$
\begin{aligned}
\rho\left(r_{n}\right) & \\
& =\inf _{v \in \Phi^{-1}\left(\left(-\infty, r_{n}\right)\right)} \frac{\sup _{u \in \Phi^{-1}\left(\left(-\infty, r_{n}\right)\right)} \Psi(u)-\Psi(v)}{r_{n}-\Phi(v)} \\
& \leq \frac{\sup _{u \in \Phi^{-1}\left(\left(-\infty, r_{n}\right)\right)} \Psi(u)-\Psi(0)}{r_{n}-\Phi(0)} \\
& =\frac{\sup _{u \in \Phi^{-1}\left(\left(-\infty, r_{n}\right)\right)} \Psi(u)}{r_{n}} \\
& \leq \frac{4 \int_{0}^{1} \max _{|u| \leq \xi_{n}} F(t, u) d t}{M_{1} \xi_{n}^{2}}
\end{aligned}
$$

which, together with (15), gives us

$$
\gamma_{1} \leq \frac{4}{M_{1}} \liminf _{\xi \rightarrow+\infty} \frac{\int_{0}^{1} \max _{|u| \leq \xi} F(t, u) d t}{\xi^{2}}=\mu_{1}<+\infty .
$$

This shows that $\left(1 / \mu_{2}, 1 / \mu_{1}\right) \subseteq\left(0,1 / \gamma_{1}\right)$. For any fixed $\lambda \in\left(1 / \mu_{2}, 1 / \mu_{1}\right)$, it follows from [22, Theorem 2.1] that either $\varphi_{\lambda}=\Phi-\lambda \Psi$ has a global minimum or there is a sequence $\left\{u_{n}\right\}$ of critical points (local minima) of $\varphi_{\lambda}$ such that $\lim _{n \rightarrow+\infty}\left\|u_{n}\right\|_{X}=+\infty$.

Next we show that the functional $\varphi_{\lambda}$ has no global minimum for $\lambda \in\left(1 / \mu_{2}, 1 / \mu_{1}\right)$. Since $\lambda>1 / \mu_{2}=$ $k_{2} / \lim \sup _{\xi \rightarrow+\infty}\left(\int_{0}^{1} F(t, \xi) d t / \xi^{2}\right)$, we can choose a constant $M_{2}$ such that, for each $n \in N=\{1,2, \ldots\}$,

$$
\sup _{\xi \geq n} \frac{\int_{0}^{1} F(t, \xi) d t}{\xi^{2}}>M_{2}>\frac{k_{2}}{\lambda}
$$

Thus, there exists $\xi_{n} \geq n$ such that

$$
\frac{\int_{0}^{1} F\left(t, \xi_{n}\right) d t}{\xi_{n}^{2}}>M_{2} .
$$

Define $u_{n}(t)$ as follows:

$$
u_{n}(t)=\xi_{n}, \quad t \in[0,1]
$$

This yields

$$
\begin{aligned}
\Phi\left(u_{n}\right) & =\frac{1}{2} k_{0} \xi_{n}^{2}+\sum_{j=1}^{m} \int_{0}^{\xi_{n}} I_{j}(s) d s \\
& \leq \frac{1}{2} k_{0} \xi_{n}^{2}+k_{1} \xi_{n}^{2} \\
& =k_{2} \xi_{n}^{2} .
\end{aligned}
$$

Then

$$
\begin{aligned}
\varphi_{\lambda}\left(u_{n}\right) & =\Phi\left(u_{n}\right)-\lambda \Psi\left(u_{n}\right) \\
& \leq k_{2} \xi_{n}^{2}-\lambda \int_{0}^{1} F\left(t, \xi_{n}\right) d t \\
& \leq \xi_{n}^{2}\left(k_{2}-\lambda M_{2}\right) .
\end{aligned}
$$

Note that $k_{2}-\lambda M_{2}<0$. Thus the functional $\varphi_{\lambda}$ has no lower bound and hence it has no global minimum and the proof is complete.

Let

$$
\begin{gathered}
\mu_{3}=\frac{4}{M_{1}} \liminf _{\omega \rightarrow 0^{+}} \frac{\int_{0}^{1} \max _{|u| \leq \omega} F(t, u) d t}{\omega^{2}}, \\
\mu_{4}=\frac{1}{k_{2}} \limsup _{\omega \rightarrow 0^{+}} \frac{\int_{0}^{1} F(t, \omega) d t}{\omega^{2}} .
\end{gathered}
$$


Theorem 7. Assume that (H3) is satisfied. If

$$
\mu_{3}<\mu_{4}
$$

holds, then, for each $\lambda \in\left(1 / \mu_{4}, 1 / \mu_{3}\right)$, problem (1) has a sequence of nonzero solutions in $X$, which weakly converges to 0 .

Proof. The proof is similar to that of Theorem 6 by showing that $\delta<+\infty$ and 0 is not a local minimum of the functional $\varphi_{\lambda}=\Phi-\lambda \Psi$.

Example 8. Consider the boundary value problem

$$
\begin{gathered}
-\left(e^{t} u^{\prime}(t)\right)^{\prime}+u(t)=\lambda f(t, u), \quad t \neq t_{1}, t \in[0,1] \\
\Delta\left(e^{t_{1}} u^{\prime}\left(t_{1}\right)\right)=u\left(t_{1}\right), \quad t_{1}=\frac{1}{2} \\
u^{\prime}(0)=u^{\prime}(1)=0
\end{gathered}
$$

where $f(t, u)=8 t u(1-\sin u)-4 t u^{2} \cos u$.

Here, $I_{1}(s)=s, p(t)=e^{t}, q(t)=1$, and $m=1$. Hence we have $k_{0}=1, k_{1}=1 / 2, k_{2}=1, M_{1}=1$, and

$$
F(t, u)=4 t(1-\sin u) u^{2}
$$

so (H3) is satisfied. Moreover, we have

$$
\begin{gathered}
\lim _{\xi \rightarrow+\infty} \inf \frac{\int_{0}^{1} \max _{|u| \leq \xi} F(t, u) d t}{\xi^{2}}=0, \\
\lim _{\xi \rightarrow+\infty} \sup \frac{\int_{0}^{1} F(t, \xi) d t}{\xi^{2}}=2 .
\end{gathered}
$$

Therefore, condition (34) holds and Theorem 6 applies: for $\lambda \in(0.5,+\infty)$, problem (47) admits an unbounded sequence of solutions in $X$.

Example 9. Consider the boundary value problem

$$
\begin{gathered}
-\left(e^{t} u^{\prime}(t)\right)^{\prime}+u(t)=\lambda f(t, u), \\
t \neq t_{1}, t \in[0,1], \\
\Delta\left(e^{t_{1}} u^{\prime}\left(t_{1}\right)\right)=u\left(t_{1}\right), \quad t_{1}=\frac{1}{2}, \\
u^{\prime}(0)=u^{\prime}(1)=0,
\end{gathered}
$$

where

$$
\begin{aligned}
f(t, u(t)) & \\
= & \left\{\begin{array}{lr}
t u\left(0.35+\frac{1}{4} \sin (\ln |u|)+\frac{1}{8} \cos (\ln |u|)\right) \\
0, \quad u=0 .
\end{array}\right.
\end{aligned}
$$

In this example, $I_{1}(s)=s, p(t)=e^{t}, q(t)=1$, and $m=1$. Hence we have $k_{0}=1, k_{1}=1 / 2, k_{2}=1, M_{1}=1$, and

$$
F(t, u(t))=\left\{\begin{aligned}
\frac{1}{2} t u^{2} & \left(0.35+\frac{1}{4} \sin (\ln |u|)\right), \\
& u \in R \backslash\{0\}, t \in[0,1], \\
0, & u=0 .
\end{aligned}\right.
$$

Hence, one has

$$
\begin{aligned}
& \frac{4}{M_{1}} \lim _{w \rightarrow 0^{+}} \inf \frac{\int_{0}^{1} \max _{|u| \leq w} F(t, u) d t}{w^{2}} \\
& \quad=0.1<\frac{1}{k_{2}} \lim _{w \rightarrow 0^{+}} \sup \frac{\int_{0}^{1} F(t, w) d t}{w^{2}}=0.15 .
\end{aligned}
$$

Therefore (46) holds. Owing to Theorem 7, when $\lambda \epsilon$ $(6.67,10)$ problem $(50)$ admits a sequence of pairwise distinct classical solutions strongly converging at 0 in $X$.

\section{Acknowledgments}

The authors are very grateful to the referees for their valuable comments and suggestions, which greatly improved the presentation of this paper. This work is supported by Hunan Provincial Natural Science Foundation of China (no. 11JJ3012).

\section{References}

[1] G. Bonanno and G. D’Aguì, "A Neumann boundary value problem for the Sturm-Liouville equation," Applied Mathematics and Computation, vol. 208, no. 2, pp. 318-327, 2009.

[2] G. Dai, "Infinitely many solutions for a Neumann-type differential inclusion problem involving the $p(x)$-Laplacian," Nonlinear Analysis: Theory, Methods \& Applications, vol. 70, no. 6, pp. 2297-2305, 2009.

[3] Y. Sun, Y. J. Cho, and D. O’Regan, “Positive solutions for singular second order Neumann boundary value problems via a cone fixed point theorem," Applied Mathematics and Computation, vol. 210, no. 1, pp. 80-86, 2009.

[4] Y. Tian and W. Ge, "The existence of solutions for a secondorder discrete Neumann problem with a p-Laplacian," Journal of Applied Mathematics and Computing, vol. 26, no. 1-2, pp. 333340, 2008.

[5] Y.-W. Zhang and H.-X. Li, "Positive solutions of a second-order Neumann boundary value problem with a parameter," Bulletin of the Australian Mathematical Society, vol. 86, no. 2, pp. 244253, 2012.

[6] D. D. Bainov and P. S. Simeonov, Impulsive Differential Equations: Periodic Solutions and Applications, Longman Scientific and Technical, Harlow, UK, 1993.

[7] V. Lakshmikantham, D. D. Baĭnov, and P. S. Simeonov, Theory of Impulsive Differential Equations, vol. 6 of Series in Modern Applied Mathematics, World Scientific Publishing, Teaneck, NJ, USA, 1989.

[8] R. P. Agarwal and D. O’Regan, "A multiplicity result for second order impulsive differential equations via the Leggett Williams fixed point theorem," Applied Mathematics and Computation, vol. 161, no. 2, pp. 433-439, 2005. 
[9] R. P. Agarwal, D. Franco, and D. O’Regan, "Singular boundary value problems for first and second order impulsive differential equations," Aequationes Mathematicae, vol. 69, no. 1-2, pp. 8396, 2005.

[10] G. Bonanno, "A critical point theorem via the Ekeland variational principle," Nonlinear Analysis: Theory, Methods \& Applications, vol. 75, no. 5, pp. 2992-3007, 2012.

[11] J. Chu and J. J. Nieto, "Impulsive periodic solutions of firstorder singular differential equations," Bulletin of the London Mathematical Society, vol. 40, no. 1, pp. 143-150, 2008.

[12] C. De Coster and P. Habets, "Upper and lower solutions in the theory of ODE boundary value problems: classical and recent results," in Non-Linear Analysis and Boundary Value Problems for Ordinary Differential Equations (Udine), F. Zanolin, Ed., vol. 371 of CISM Courses and Lectures, pp. 1-78, Springer, Vienna, Austria, 1996.

[13] M. Galewski and S. Głab, "On the discrete boundary value problem for anisotropic equation," Journal of Mathematical Analysis and Applications, vol. 386, no. 2, pp. 956-965, 2012.

[14] J. J. Nieto and D. O’Regan, "Variational approach to impulsive differential equations," Nonlinear Analysis: Real World Applications, vol. 10, no. 2, pp. 680-690, 2009.

[15] D. Qian and X. Li, "Periodic solutions for ordinary differential equations with sublinear impulsive effects," Journal of Mathematical Analysis and Applications, vol. 303, no. 1, pp. 288-303, 2005.

[16] J. Shen and W. Wang, "Impulsive boundary value problems with nonlinear boundary conditions," Nonlinear Analysis: Theory, Methods \& Applications, vol. 69, no. 11, pp. 4055-4062, 2008.

[17] Y. Tian and W. Ge, "Applications of variational methods to boundary-value problem for impulsive differential equations," Proceedings of the Edinburgh Mathematical Society. Series II, vol. 51, no. 2, pp. 509-527, 2008.

[18] H. Zhang and Z. Li, "Variational approach to impulsive differential equations with periodic boundary conditions," Nonlinear Analysis: Real World Applications, vol. 11, no. 1, pp. 67-78, 2010.

[19] X. Zhang and X. Tang, "Subharmonic solutions for a class of non-quadratic second order Hamiltonian systems," Nonlinear Analysis: Real World Applications, vol. 13, no. 1, pp. 113-130, 2012.

[20] Z. Zhang and R. Yuan, "An application of variational methods to Dirichlet boundary value problem with impulses," Nonlinear Analysis: Real World Applications, vol.11, no. 1, pp. 155-162, 2010.

[21] G. Bonanno and P. Candito, "Non-differentiable functionals and applications to elliptic problems with discontinuous nonlinearities," Journal of Differential Equations, vol. 244, no. 12, pp. 3031-3059, 2008.

[22] G. Bonanno and G. M. Bisci, "Infinitely many solutions for a boundary value problem with discontinuous nonlinearities," Boundary Value Problems, vol. 2009, Article ID 670675, 20 pages, 2009. 


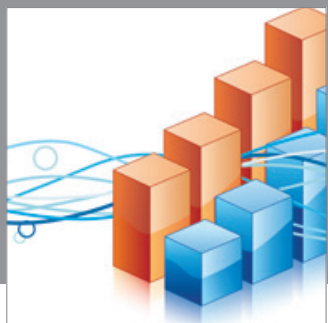

Advances in

Operations Research

mansans

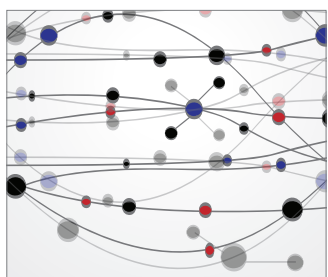

The Scientific World Journal
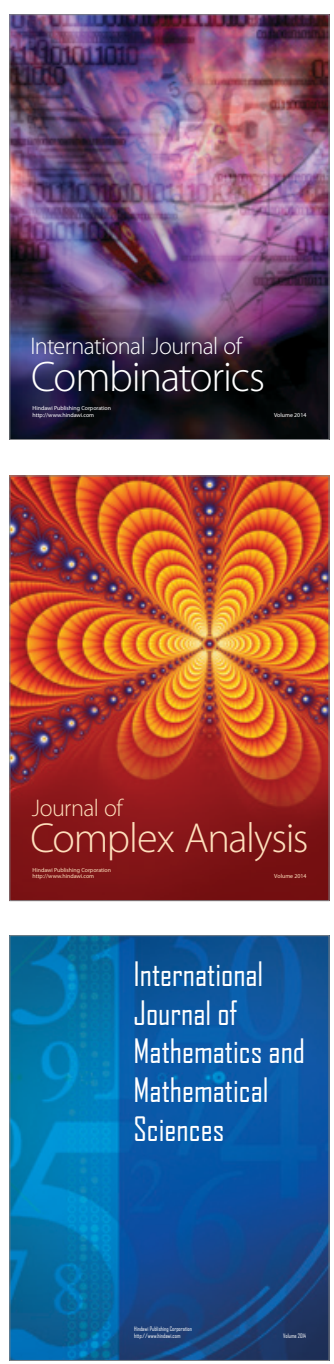
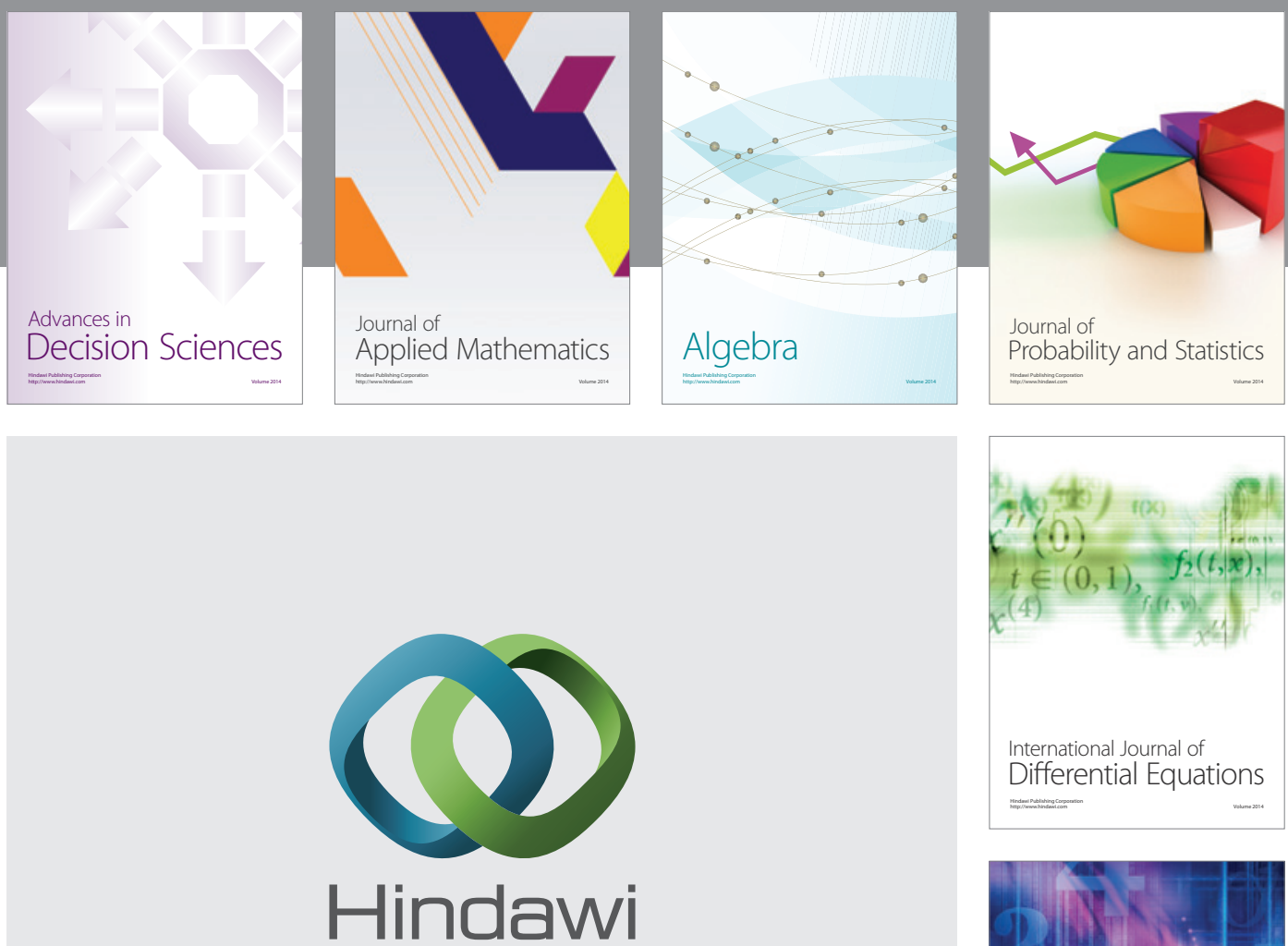

Submit your manuscripts at http://www.hindawi.com
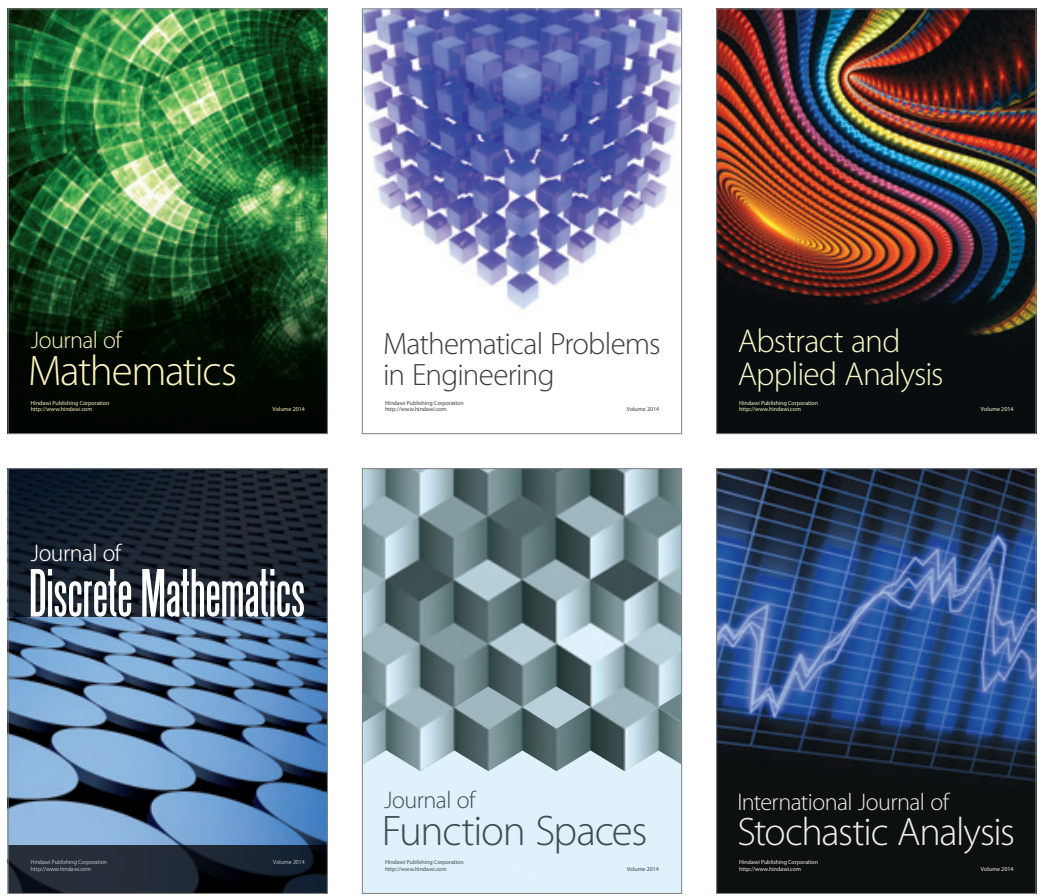

Journal of

Function Spaces

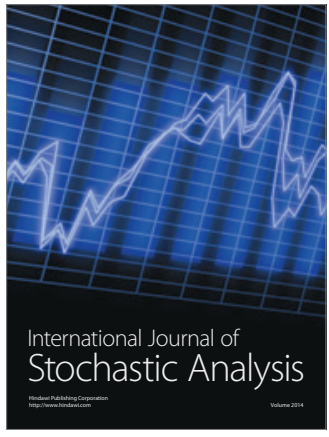

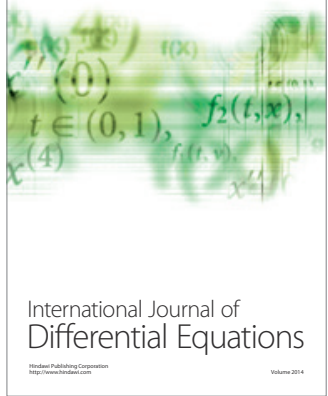
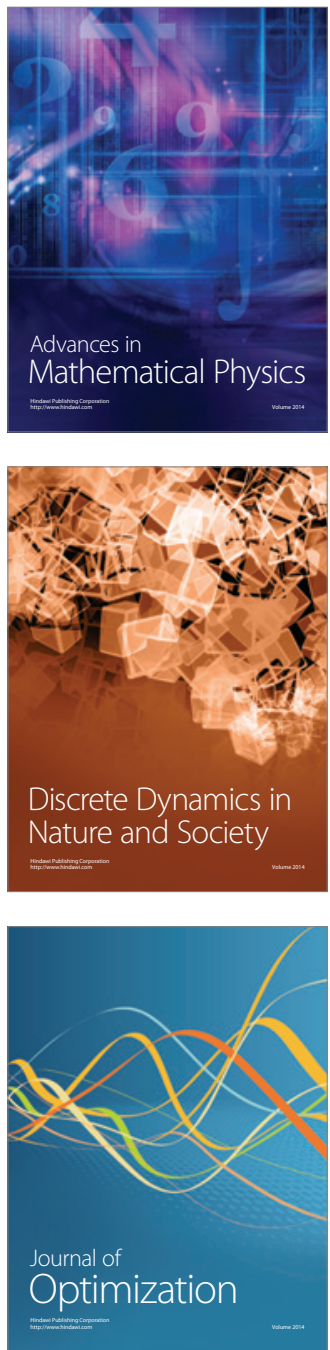